Polymer Journal, Vol. 39, No. 7, pp. 684-689 (2007)

(C) 2007 The Society of Polymer Science, Japan

\title{
Effects of Static and Dynamic Forces on Surface Viscoelastic Response of Polymer Films in Scanning Viscoelasticity Microscopy
}

\author{
Kei-ichi AKabori, ${ }^{1}$ Keiji TAnAKa,${ }^{1, \dagger}$ Noriaki SATOMI, ${ }^{1}$ Toshihiko NAgamura, ${ }^{1}$ \\ Atsushi TAKAHARA, ${ }^{2, \dagger}$ and Tisato KAJIYAMA ${ }^{3}$ \\ ${ }^{1}$ Department of Applied Chemistry, Kyushu University, 744 Motooka, Nishi-ku, Fukuoka 819-0395, Japan \\ ${ }^{2}$ Institute for Materials Chemistry and Engineering, Kyushu University, \\ 6-10-1 Hakozaki, Higashi-ku, Fukuoka 812-8581, Japan \\ ${ }^{3}$ Kyushu University, 6-10-1 Hakozaki, Higashi-ku, Fukuoka 812-8581, Japan
}

(Received February 5, 2007; Accepted April 4, 2007; Published May 16, 2007)

\begin{abstract}
Scanning force microscopy with force-modulation mode enables one to gain direct insights into local viscoelastic properties at polymer surfaces. Changing modulation condition such as dynamic amplitude and frequency in addition to static normal force, surface viscoelastic response of polystyrene (PS) film was studied. If dynamic and/or static forces were/was ill-chosen, non-linearity of viscoelastic response became dominant, resulting in hole formation at the surface. On the contrary, if they were appropriately adjusted, linear viscoelastic measurement could be realized in the frequency range from $10 \mathrm{~Hz}$ to $4 \mathrm{kHz}$. This leads to traditional rheological analysis even at the surface.

[doi:10.1295/polymj.PJ2006230]

KEY WORDS Scanning Force Microscopy / Surface / Glass Transition / Polystyrene / Rheological Analysis /
\end{abstract}

So far, it has been believed that polymer materials should be as small as possible to design and construct highly-assembled organic devices. Actually, it is not technically so difficult to prepare such tiny polymeric pieces with the advent of modern nanotechnology. However, the problem is that once the pieces become smaller than approximately $100 \mathrm{~nm}$, their physical properties would be altered from the corresponding bulk behaviors mainly due to a large surface ratio to the total volume. ${ }^{1}$ This means that the reliability and durability of the devices cannot be simply deduced on the basis of the bulk properties of each polymer component.

Scanning force microscopy (SFM) has been widely used to examine surface morphology and properties for various kinds of materials. ${ }^{2,3}$ Restricting ourselves to studies on surface rheology in polymer films, various types of SFM such as lateral force microscopy (LFM), ${ }^{4-8}$ tapping mode atomic force microscopy (TM-AFM), ${ }^{9-12}$ and force volume measurement ${ }^{13-15}$ have been developed. SFM with the force modulation mode (FM-SFM) also possesses an intriguing potential to obtain local viscoelasticity at the surface of polymers. ${ }^{2,3,16-26}$ Of FM-SFM, two alternative modes of X-modulation ${ }^{16-19}$ and Z-modulation, ${ }^{20-26}$ depending on whether the modulation direction is lateral or normal to the sample surface, have been proposed. We call the latter technique as scanning viscoelasticity microscopy (SVM) ${ }^{23}$ Since the principle of SVM is basically the same as the traditional dynamic mechanical analysis (DMA), it is relatively easy, compared with other techniques, to consider what information obtained means. Although we have already published some results for surface viscoelastic properties of $\mathrm{PS},{ }^{23}$ it is still open what happens if the tip modulation is made under various conditions.

In this study, an effect of tip modulation manner on viscoelastic response at the surface of a polystyrene (PS) film is systematically studied. After one of better modulation conditions for SVM is adopted, rheological analysis is applied to the surface viscoelasticity of the PS. We believe that SVM will not be generalized without the results after this study.

\section{EXPERIMENTAL}

A polymer used in this study was monodisperse PS, which was synthesized by an anionic living polymerization. The values of number-average molecular weight $\left(M_{\mathrm{n}}\right)$ and molecular weight distribution $\left(M_{\mathrm{w}} / M_{\mathrm{n}}\right)$, where $M_{\mathrm{w}}$ denotes weight-average molecular weight, were $140 \mathrm{k}$ and 1.05 , respectively. The bulk glass transition temperature $\left(T_{\mathrm{g}} \mathrm{b}\right)$ measured by differential scanning calorimetry (DSC) was $382 \mathrm{~K}$. A PS film was spin-coated from a toluene solution onto a cleaned silicon $(\mathrm{Si})$ substrate with a native oxide

${ }^{\dagger}$ To whom correspondence should be addressed (Tel: +81-92-802-2879 (KT), +81-92-642-2721 (AT), Fax: +81-92- 802-2880 (KT), +81-92-642-2715 (AT), E-mail: k-tanaka@cstf.kyushu-u.ac.jp (KT), takahara@cstf.kyushu-u.ac.jp (AT)). 
layer. To remove residual solvent molecules and to eliminate stress imposed by the preparation procedure, the PS film was dried overnight at room temperature, and then was annealed at $423 \mathrm{~K}$ above the $T_{\mathrm{g}}{ }^{\mathrm{b}}$ for, at least $24 \mathrm{~h}$ in a vacuum oven. After the annealing, the film was cooled to room temperature at the rate of about $0.5 \mathrm{~K} \mathrm{~min}^{-1}$. The film thickness evaluated by ellipsometric measurement was approximately $200 \mathrm{~nm}$, which was sufficient to avoid any ultrathinning effects on surface relaxation behavior. ${ }^{27}$ Using an atomic force microscope (AFM), we confirmed that the film surface was quite smooth with the roughness being less than $0.5 \mathrm{~nm}$.

SVM measurements were conducted by an SPA300HV with an SPI3800 controller (SIINT Inc.) under vacuum. The cantilevers used were purchased from Olympus Co. Ltd. and were characterized by the following procedures. The spring constant was determined on the basis of the resonant frequencies before and after the deposition of a gold thin layer on it. ${ }^{28}$ And, the tip radius was measured using colloidal gold particles with known size. ${ }^{29}$ The values so obtained were $0.86 \pm 0.07 \mathrm{~N} \mathrm{~m}^{-1}$ and $14.2 \pm 1.2 \mathrm{~nm}$, respectively. A static normal force $\left(F_{\mathrm{N}}\right)$, which was imposed after approaching a tip, was set to be from 1 to $20 \mathrm{nN}$. In addition to $F_{\mathrm{N}}$, the tip was sinusoidaly modulated at a fixed position during the SVM measurements. The amplitude $(A)$ and frequency $(f)$ of the modulation were precisely controlled by means of an external function generator and a piezoelectric element as an electro-mechanical transducer. The response force signal, which basically reflects surface viscoelasticity of the sample, was simultaneously analyzed together with the imposed modulation signal by a lock-in amplifier via GP-IB interface. To evaluate the surface relaxation behavior in the PS film, the phase lag between the imposed and the response signals $\left(\delta^{\mathrm{s}}\right)$ was measured as a function of temperature. For direct comparison between the surface and the bulk data, we also conducted a traditional DMA of a bulk PS with a comparable $M_{\mathrm{n}} \cdot{ }^{27}$

\section{RESULTS AND DISCUSSION}

In general, DMA should be made in a linear viscoelastic regime. If applied strain exceeds a certain value, the sample is suffered from plastic deformation, that is, structural destruction. In the bulk DMA measurement, the border between linear and non-linear regions can be easily judged on the basis of Lissajous figure, which basically corresponds to the stress-strain curve. On the other hand, in SVM measurement, a tip is supposed to be penetrated to the sample surface under a force. Then, the tip is sinusoidaly modulated with an amplitude, resulting in that force modulation

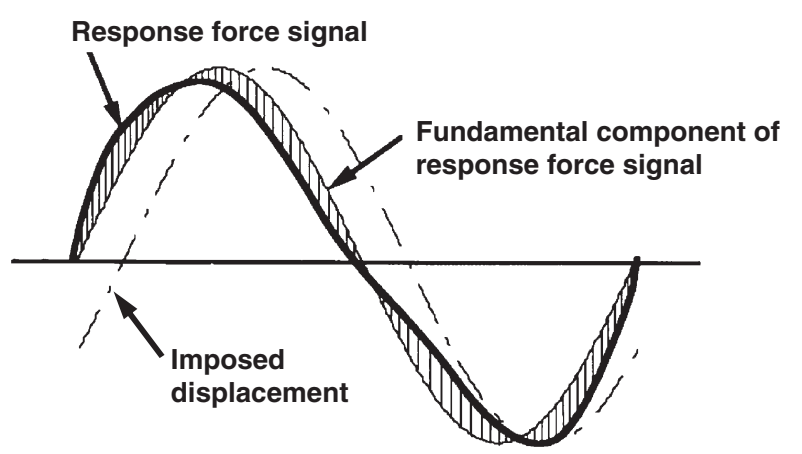

Figure 1. A schematic representation for the non-linear viscoelastic response.

is applied to the sample surface in addition to the static force. Actually, this is the origin of the name for the method. We call the former and latter static and dynamic forces, respectively.

First of all, in order to seek appropriate forces corresponding to a linear response region, the wave analysis for the response force signal was made in conjunction with the morphological observation by AFM. In this analysis, nonlinear viscoelastic parameter (NVP), which was defined as a fraction of harmonic wave components to the first one, was used as an index for nonlinearity. ${ }^{30}$ Figure 1 shows the schematic representation for the non-linear viscoelastic response. The NVP is formulated by eqs 1 and 2 .

$$
\begin{gathered}
d(t)=d_{1} \sin \left(\omega \mathrm{t}+\delta_{1}\right)+d_{2} \sin \left(2 \omega \mathrm{t}+\delta_{2}\right)+\ldots \\
+d_{\mathrm{n}} \sin \left(\mathrm{n} \omega \mathrm{t}+\delta_{\mathrm{n}}\right) \\
\mathrm{NVP}=\left(d_{2}+d_{3}+\ldots+d_{\mathrm{n}}\right) / d_{1}
\end{gathered}
$$

Here, $d$ and $\delta$ are amplitude of response force signal and phase lag, respectively. Figure 2 shows the relation of NVP value to $A$ as a function of $F_{\mathrm{N}}$ for PS films and $\mathrm{Si}$ wafers, which are specified as labels 1 and 2, respectively. Since the modulation was driven using a bimorph element equipped at the end of the cantilever, it should be noted that the actual response amplitude of the tip was supposed to be smaller than the A. Actually, it was possible to evaluate directly the response amplitude. However, experimental independent was the $A$ value and thus the response amplitude could not be precisely controlled. This was because it also depended on the $F_{\mathrm{N}}$ value. Assuming that modulus and Poisson's ratio of the PS are equivalent to their bulk values, the maximum static pressure applied to the surface $(P)$ after the tip approach can be calculated on the basis of Hertzian elastic model. ${ }^{31}$ Since the tip radius was $14.2 \mathrm{~nm}$, the $P$ values were simply calculated to be 490,620 and $780 \mathrm{MPa}$ for the panel (a), (b) and (c), respectively. As a general trend, the NVP increased with increasing $A$, meaning that non-linearity of the response force signal became 

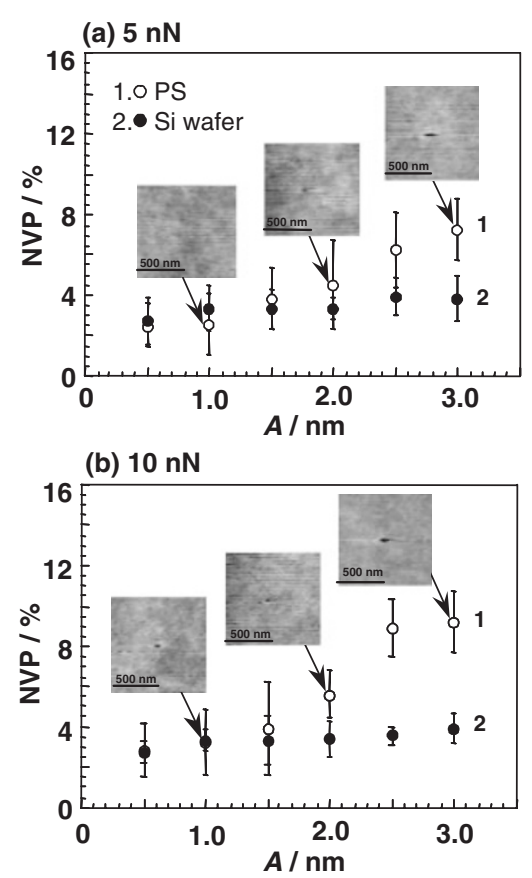

(c) $20 \mathrm{nN}$

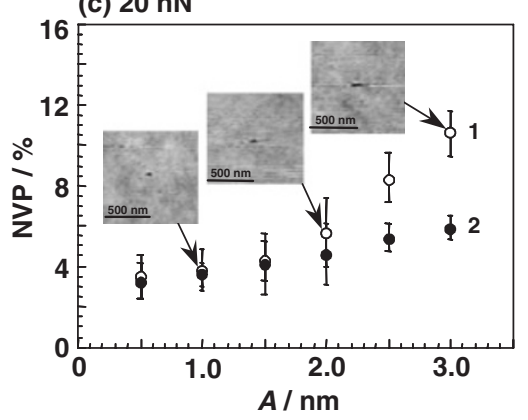

Figure 2. Relation between modulation amplitude of the cantilever $(A)$ and non-linear viscoelastic parameter (NVP) as a function of $F_{\mathrm{N}}$ for PS films and Si wafers specified as labels 1 and 2, respectively. The inset images are topographical ones after SVM measurements.

dominant with increasing $A$. The $\mathrm{Si}$ wafer is not viscoelastic but elastic at room temperature, and possesses Young's modulus of $280 \mathrm{GPa}$, being much harder than the cantilever. Nevertheless, the NVP for the Si surface was always finite under conditions employed and increased with increasing $A$, especially, larger than $1.5 \mathrm{~nm}$. Such the non-linear response from the $\mathrm{Si}$ surface might be arisen from a situation that another motion of the tip, like a slipping motion, would occur at the contact point due to the hardness of the wafer. This factor might be less important for the PS surface because of its softness compared with the $\mathrm{Si}$ wafer. In the case of the $A$ smaller than $1.0 \mathrm{~nm}$, the NVP value was similar in the two surfaces. However, once the $A$ went beyond 1.5 or $2.0 \mathrm{~nm}$, the NVP for the PS surface started to be larger than that for the Si. This fashion itself seems to be insensitive to the $F_{\mathrm{N}}$. To address whether the non-linearity observed for the PS surface was assigned to surface
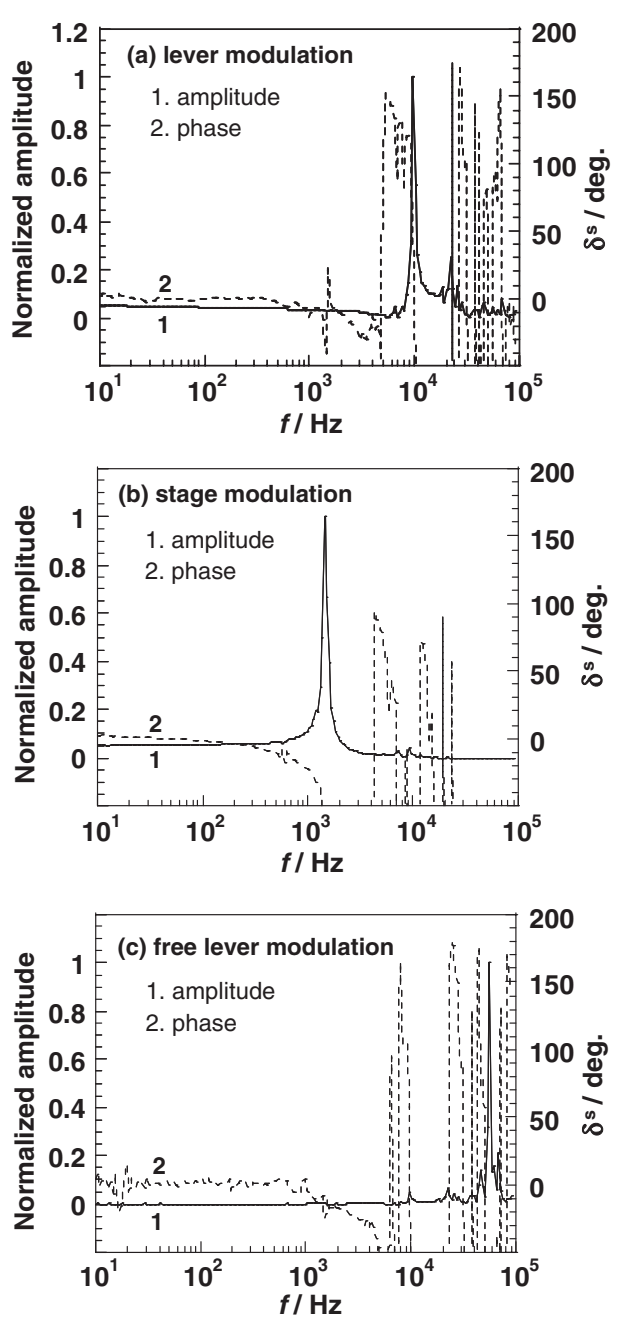

Figure 3. Frequency dependence of normalized amplitude of response force signal and phase lag between the imposed and the response signals for (a) lever modulation, (b) stage modulation, (c) free lever modulation, under contacting a tip with a Si wafer.

plastic deformation, surface morphology of the PS films was observed by AFM, as shown in the insets of each panel of Figure 2. When the $A$ was larger than $2.0 \mathrm{~nm}$, a hole was artificially made at the PS surface. If the $F_{\mathrm{N}}$ was higher than $10 \mathrm{nN}$, a hole was made even at the $A$ of $1.0 \mathrm{~nm}$. On the contrary, it was confirmed that the PS surface was not damaged under the $F_{\mathrm{N}}$ of $10 \mathrm{nN}$ for LFM measurements, ${ }^{5}$ implying that the additional dynamic force induces the hole formation. Our previous works published have been carried out within the non-destructive linear viscoelastic region. ${ }^{23}$

So far, we have examined surface molecular motion of polymer films by SVM in the frequency range of a decade. ${ }^{23}$ If the range is enlarged, at least to be 2 decades, rheological analysis at the surface becomes possible. For the purpose, the frequency spectra for our experimental set-up should be obtained at first. Figure 3 shows the relation between frequency and 
modulation amplitude of a lever. Here, the modulation amplitude corresponds to not imposed signal but response one. The ordinate was normalized by a maximum value. Also, $\delta^{\mathrm{s}}$ is shown as a function of frequency. To drive the force modulation, there are two ways; (a) lever modulation and (b) stage modulation. The former and latter were carried out by modulating a cantilever and a sample stage, respectively. The both were made under a condition that a tip contacted with the Si surface. To eliminate a convolution from the feedback system of SVM, the circuit controller for the feedback loop was turned off during data acquisition. For comparison, the data for free lever modulation is also shown in the panel (c). In the case of the free modulation, a strong peak was observed at the resonant frequency of $56.4 \mathrm{kHz}$, and small peaks were accompanied at lower and higher sides of the resonant frequency. When a cantilever tip was modulated and contacted with the $\mathrm{Si}$ substrate, the largest peak was shifted to be $9.6 \mathrm{kHz}$, as shown in the panel (a) of Figure 3. This was simply because the vibration system was altered and thus the resonant frequency was concurrently shifted to the lower side. Modulating the sample stage under the contact with the cantilever tip, the lever amplitude was maximized at around $1.4 \mathrm{kHz}$, as seen in the panel (b) of Figure 3. This would be arisen from the resonance of the sample stage, because the frequency was changed with changing size of the stage. However, it was clear that the modulation was quite stable at frequencies lower than the resonant value. Although the phase data was a little fluctuated in comparison with the amplitude one, it was experimentally confirmed that $\delta^{s}$ measured at the Si wafer was independent of measuring temperature at a given frequency. On the other hand, the $\delta^{\mathrm{s}}$ value prominently changed once polymer surface reached glass transition, ${ }^{23}$ indicating that it

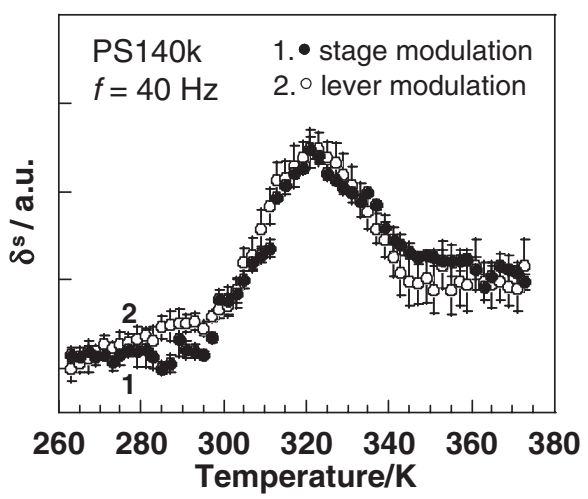

Figure 4. Temperature dependence of surface phase lag for a PS140k film at the frequency of $40 \mathrm{~Hz}$. Filled and open circles are corresponding to the data acquired by stage and lever modulation SVM, respectively.

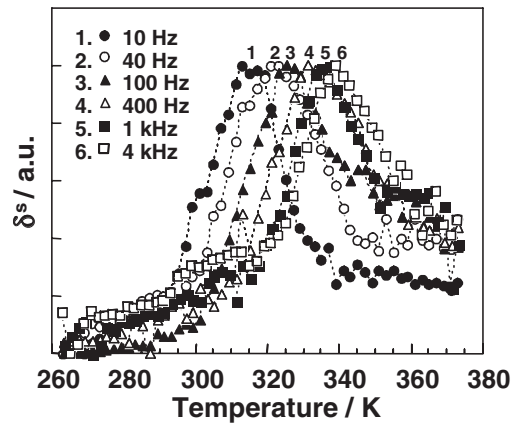

Figure 5. Temperature dependence of surface phase lag for the PS140k film as a function of frequency $(f)$ with cantilever modulation SVM.

was quite sensitive to viscoelastic response of the polymer surface.

Figure 4 shows the temperature dependence of $\delta^{\mathrm{s}}$ for a PS140k film at the frequency of $40 \mathrm{~Hz}$. This frequency is quite comparable with that for bulk DMA. Filled and open circles were obtained by the stage and lever modulations, respectively. In both cases, a peak corresponding to the surface $\alpha_{\mathrm{a}}$-relaxation process was clearly observed at around $320 \mathrm{~K} .^{5,23}$ Also, the peak shape and position were quite similar each other. Thus, as long as the surface relaxation is discussed on the basis of temperature dependence of $\delta^{\mathrm{s}}$, it is not so important which a driving method, stage or lever modulation, is chosen. However, as already shown in Figure 3, it seems that the cantilever modulation was more stable than the stage modulation over the wide frequency range up to several $\mathrm{kHz}$. Thus, the cantilever modulation was adopted hereafter. In addition, the $A$ and the $F_{\mathrm{N}}$ values were fixed to be $1 \mathrm{~nm}$ and $1 \mathrm{nN}$, respectively, which were firmly assured within the nondestructive region, as shown in Figure 2.

Figure 5 shows the temperature dependence of $\delta^{\mathrm{s}}$ as a function of $f$ for the PS140k film. The $f$ was truncated at $4 \mathrm{kHz}$ because the SVM system was not stable at higher $f$, as shown in the panel (a) of Figure 3. The peak position shifted toward a higher temperature with increasing $f$, indicating that the peak was arisen from a relaxation process. Here, we make a direct comparison between surface and bulk DMA data. The peak temperature obtained by SVM at the frequency of $10 \mathrm{~Hz}$ was lower than that by DMA at the frequency of $11 \mathrm{~Hz}$, and the difference was more than $60 \mathrm{~K}$. This result makes it clear that the $T_{\mathrm{g}}{ }^{\mathrm{s}}$ was definitely lower than the $T_{\mathrm{g}} \mathrm{b}$. The $T_{\mathrm{g}}$ depression at the surface was in good agreement with the reported result by another methods. ${ }^{32-41}$

To analyze the segmental dynamics, the relation between the reciprocal number of $T_{\max }$ and $\ln \tau$ was plotted in Figure 6. Here, $T_{\max }$ and $\tau$ are temperature, 


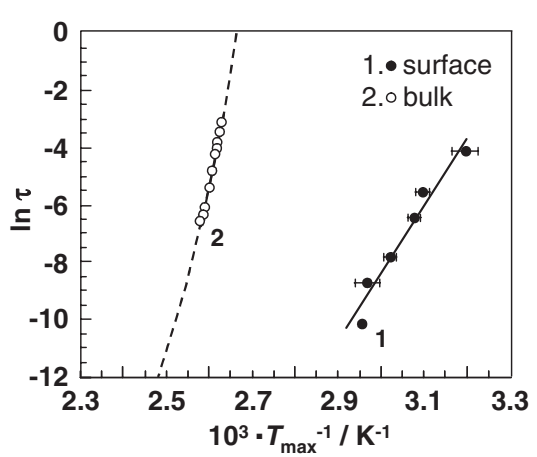

Figure 6. Relationship between reciprocal peak temperature $\left(1 / T_{\max }\right)$ and $\ln \tau$ for the surface $\alpha_{\mathrm{a}}$-relaxation in the PS140k film. The data for the bulk PS is also plotted in the figure. The dotted curve denotes the prediction based on Vogel-Fulcher equation using bulk parameters.

at which $\delta^{s}$ was maximized, and relaxation time for the segmental motion, respectively. The $\tau$ was simply calculated by $(1 / 2 \pi f)$. The dotted curve in Figure 6 denotes the prediction based on Vogel-Fulcher (VF) equation; ${ }^{42}$

$$
\tau=\tau_{0} \exp \left\{B /\left(T-T_{\mathrm{V}}\right)\right\}
$$

where $\tau_{0}, T_{\mathrm{v}}$ and $B$ are characteristic time related to the molecular vibration, Vogel temperature and activation temperature, respectively. Using $T_{\mathrm{V}}$ of $324 \mathrm{~K}$ and $B$ of $1733 \mathrm{~K},{ }^{43}$ the experimental data for the bulk PS was fitted with $\tau_{0}$ as a parameter. On the other hand, the trial was not applied to the surface. This was because the time domain for the relaxation process was only within two decades, leading to the difficulty in reliable fitting with three independent parameters such as $\tau_{0}, T_{\mathrm{v}}$ and $B$. However, it is apparent that the data set for the surface relaxation was shifted to the lower temperature (higher $1 / T_{\max }$ ) side at a given $\tau$ and that the slope of the relation became less at the surface. These imply that the surface molecular motion is much more enhanced than the bulk one.

Looking at Figure 6, it can be assumed that the relation between $1 / T_{\max }$ and $\ln \tau$ is linear within the range employed. This is more realistic for the surface. Hence, Arrhenius analysis was applied to the experimental data. The apparent activation energy $\left(\Delta H^{\ddagger}\right)$ for the relaxation process is given by

$$
\Delta H^{\ddagger}=R_{\text {gas }} \mathrm{d}(\ln \tau) / \mathrm{d}\left(1 / T_{\max }\right)
$$

where $R_{\text {gas }}$ is gas constant. The surface $\Delta H^{\ddagger}$ value obtained on the basis of the linear slope in the figure was approximately $200 \pm 20 \mathrm{~kJ} \mathrm{~mol}^{-1}$, which was in good agreement with the previous value evaluated by LFM. ${ }^{5}$ Comparing this number with the bulk $\Delta H^{\ddagger}$ value of $610 \pm 15 \mathrm{~kJ} \mathrm{~mol}^{-1}$, it is clear that the segments existed in the proximity to the surface can move easier than those in the internal bulk due to the lack of restriction from the air side. ${ }^{44}$ This should be one of reasons why $T_{\mathrm{g}} \mathrm{s}$ was lower than the $T_{\mathrm{g}} \mathrm{b}$.

\section{CONCLUSIONS}

Effects of modulation manner on surface viscoelastic response of PS in SVM were demonstrated. When the dynamic and static forces applied to the surface exceeded critical values, non-linearity of surface viscoelasiticity in the PS film became dominant, resulting in the plastic surface deformation. On the contrary, if those conditions were carefully chosen, linear viscoelastic measurements were possible in the frequency range more than 2 decades. Comparing the SVM data with the bulk DMA results, it was claimed that both the transition temperature and the $\Delta H^{*}$ of the surface segmental motion for the PS were lower than the corresponding bulk values.

Acknowledgment. This research was partly supported by Industrial Technology Research Grant Program in 2006 from New Energy and Industrial Technology Development Organization (NEDO) of Japan, and the Grant-in-Aids for Young Scientists A (No. 18685014) and for the Science Research in a Priority Area "Soft Mather Physics" (No. 19031021) from Ministry of Education, Culture, Sports, Science and Technology, Japan.

\section{REFERENCES}

1. J. A. Forrest and R. A. L. Jones, "Polymer Surfaces, Interfaces and Thin Films," A. Karim and S. Kumar, Ed., World Scientific, Singapore, 2000, and references therein.

2. "Scanning Probe Microscopy of Polymers," B. D. Ratner and V. V. Tsukuruk, Ed., ACS Symp. Ser., Washington, DC, 1998 .

3. "Procedure in Scanning Probe Microscopies," 1st ed., R. J. Colton, A. Engel, J. E. Frommer, H. E. Gaub, A. Gewirth, R. Guckenberger, J. Rabe, W. M. Heckl, and B. Parkinson, Ed., John Wiley \& Sons, 1998.

4. a) G. Haugstad and W. L. Gladfelter, Langmuir, 11, 3473 (1995).

b) J. A. Hammerschmidt, B. Moasser, W. L. Gladfelter, G. Haugstad, and R. R. Jones, Macromolecules, 29, 8996 (1996).

c) J. A. Hammerschmidt, W. L. Gladfelter, and G. Haugstad, Macromolecules, 32, 3360 (1999).

d) R. H. Schmidt, G. Haugstad, and W. L. Gladfelter, Langmuir, 19, 10390 (2003).

5. a) T. Kajiyama, K. Tanaka, and A. Takahara, Macromolecules, 30, 280 (1997).

b) K. Tanaka, A. Takahara, and T. Kajiyama, Macromolecules, 33, 7588 (2000).

c) K. Tanaka, T. Kajiyama, A. Takahara, and S. Tasaki, Macromolecules, 35, 4702 (2002).

d) A. Sakai, K. Tanaka, Y. Fujii, T. Nagamura, and T. 
Surface Viscoelastic Response of Polymer Films

Kajiyama, Polymer, 46, 429 (2005).

6. a) F. Dinelli, C. Buenviaje, and R. M. Overney, J. Chem. Phys., 113, 2043 (2000).

b) F. Dinelli, C. Buenviaje, and R. M. Overney, Thin Solid Films, 396, 138 (2001).

c) S. Sills, T. Gray, and R. M. Overney, J. Chem. Phys., 123, 134902 (2005).

7. J. Fu, B. Li, and Y. Han, J. Chem. Phys., 123, 064713 (2005).

8. M. Komura, Z. B. Qiu, T. Ikehara, K. Nakajima, and T. Nishi, Polym. J., 38, 31 (2006).

9. a) G. Bar, R. Brandsch, and M. H. Whangbo, Langmuir, 14, 7343 (1998).

b) G. Bar, L. Delineau, R. Brandsch, M. Bruch, and M. H. Whangbo, Appl. Phys. Lett., 75, 4198 (1999).

c) G. Bar, M. Ganter, R. Brandsch, L. Delineau, and M. H. Whangbo, Langmuir, 16, 5702 (2000).

10. X. Chen, M. C. Davies, C. J. Roberts, S. J. B. Tendler, P. M. Williams, J. Davies, A. C. Dawkes, and J. C. Edwards, Ultramicroscopy, 75, 171 (1998).

11. H. Bodiguel, H. Montes, and C. Fretigny, Rev. Sci. Instrum., 75, 2529 (2004).

12. W. Xu, P. M. Wood-Adams, and C. G. Robertson, Polymer, 47, 4798 (2006).

13. C. Reynaud, F. Sommer, C. Quet, N. E. Bounia, and T. M. Duc, Surf. Interface Anal., 30, 185 (2000).

14. M. Lemieux, D. Usov, S. Minko, M. Stamm, H. Shulha, and V. V. Tsukruk, Macromolecules, 36, 7244 (2003).

15. H. Nukaga, S. Fujinami, H. Watabe, K. Nakajima, and T. Nishi, Jpn. J. Appl. Phys., 44, 5425 (2005).

16. K. J. Wahl, S. V. Stepnowski, and W. N. Unertl, Trib. Lett., 5, 103 (1998).

17. P. E. Mazeran and J. L. Loubet, Trib. Lett., 7, 199 (1999).

18. a) S. Ge, Y. Pu, W. Zhang, M. Rafailovich, J. Sokolov, C. Buenviaje, R. Buckmaster, and R. M. Overney, Phys. Rev. Lett., 85, 2340 (2000).

b) Y. Pu, S. R. Ge, M. Rafailovich, J. Sokolov, Y. Duan, E. Pearce, V. Zaitsev, and S. Schwarz, Langmuir, 17, 5865 (2001).

c) Y. Ji, B. Q. Li, S. R. Ge, J. C. Sokolov, and M. H. Rafailovich, Langmuir, 22, 1321 (2006).

19. S. Moon and M. D. Foster, Langmuir, 18, 1865 (2002).

20. P. Maivald, H. J. Butt, S. A. C. Gould, C. B. Prater, B. Drake, J. A. Gurley, V. B. Elings, and P. K. Hansma, Nanotechnology, 2, 103 (1991).

21. a) N. A. Burnham, R. J. Colton, and H. M. Pollock, Nanotechnology, 4, 64 (1993).

b) F. Oulevey, G. Gremaud, A. Semoroz, A. J. Kulik, N. A. Burnham, E. Dupas, and D. Gourdon, Rev. Sci. Instrum., 69, 2085 (1998).

c) F. Oulevey, N. A. Burnham, G. Gremaud, A. J. Kulik, H. M. Pollock, A. Hammiche, M. Reading, M. Song, and D. J. Hourston, Polymer, 41, 3087 (2000).
22. M. Radmacher, R. W. Tillmann, and H. E. Gaub, Biophys. J., 64, 735 (1993).

23. a) T. Kajiyama, K. Tanaka, I. Ohki, S.-R. Ge, J.-S. Yoon, and A. Takahara, Macromolecules, 27, 7932 (1994).

b) K. Tanaka, A. Taura, S.-R. Ge, A. Takahara, and T. Kajiyama, Macromolecules, 29, 3040 (1996).

c) N. Satomi, A. Takahara, and T. Kajiyama, Macromolecules, 32, 4474 (1999).

d) K. Tanaka, K. Hashimoto, A. Takahara, and T. Kajiyama, Langmuir, 19, 6573 (2003).

24. E. W. Stroup, A. Pungor, and V. Hlady, Ultramicroscopy, 66, 237 (1996).

25. D. DeVecchio and B. Bhushan, Rev. Sci. Instrum., 68, 4498 (1997).

26. P. E. Mazeran and J. L. Loubet, Trib. Lett., 3, 125 (1997).

27. K. Akabori, K. Tanaka, T. Nagamura, A. Takahara, and T. Kajiyama, Macromolecules, 38, 9735 (2005).

28. C. T. Gibson, B. L. Weeks, J. R. E. Lee, C. Abell, and T. Rayment, Rev. Sci. Instrum., 72, 2340 (2001).

29. J. Vesenka, S. Manne, R. Giberson, T. Marsh, and E. Henderson, Biophys. J., 65, 992 (1993).

30. a) N. J. Jo, A. Takahara, and T. Kajiyama, Polym. J., 25, 721 (1993).

b) T. Liang, A. Tokunaga, A. Yamashita, A. Takahara, and T. Kajiyama, Polym. Bull., 36, 477 (1996).

31. “Contact Mechanics," K. L. Johnson, Ed., Cambridge University Press, Cambridge, 1999.

32. Y. C. Jean, R. Zhang, H. Cao, J. P. Yuan, C. M. Huang, B. Nielsen, and P. Asoka-Kumar, Phys. Rev. B: Condens. Matter Mater. Phys., 56, R8459 (1997).

33. Y. M. Boiko and R. E. Prud'homme, J. Polym. Sci., Part B: Polym. Phys., 36, 567 (1998).

34. A. D. Schwab, D. M. G. Agra, J. H. Kim, S. Kumar, and A. Dhinojwala, Macromolecules, 33, 4903 (2000).

35. V. Zaporojtchenko, T. Strunskus, J. Erichsen, and F. Faupel, Macromolecules, 34, 1125 (2001).

36. D. Kawaguchi, K. Tanaka, A. Takahara, and T. Kajiyama, Macromolecules, 34, 6164 (2001).

37. V. N. Bliznyuk, H. E. Assender, and G. A. D. Briggs, Macromolecules, 35, 6613 (2002).

38. H. Fischer, Macromolecules, 35, 3592 (2002).

39. J. H. Teichroeb and J. A. Forrest, Phys. Rev. Lett., 91, 016104 (2003).

40. R. Weber, I. Grotkopp, J. Stettner, M. Tolan, and W. Press, Macromolecules, 36, 9100 (2003).

41. H. Morita, K. Tanaka, T. Kajiyama, T. Nishi, and M. Doi, Macromolecules, 39, 6233 (2006).

42. a) H. Vogel, Phys. Z, 22, 645 (1921).

b) G. S. Fulcher, J. Am. Ceram. Soc., 77, 3701 (1925).

43. K. Fukao and Y. Miyamoto, Phys. Rev. E, 64, 011803 (2001).

44. K. L. Ngai, A. K. Rizos, and D. J. Plazek, J. Non-Cryst. Solids, 235, 435 (1998). 